\title{
Nano-Encapsulated Smart Tunable Phase Change
}

\author{
Materials \\ S. Sinha-Ray ${ }^{a}$, R.P. Sahu ${ }^{a}$, A.L. Yarin*a,b \\ ${ }^{a}$ Department of Mechanical and Industrial Engineering, \\ University of Illinois at Chicago, \\ 842 W. Taylor St., Chicago IL 60607-7022, USA. Tel: (312) 996-3472 \\ E-mail: ayarin@uic.edu \\ ${ }^{\mathrm{b}}$ Center for Smart Interfaces, Technische Universität Darmstadt
}

Petersenstr. 32, 64287 Darmstadt, Germany

We achieve the intercalation of different types of paraffins and their mixtures with triglycerides inside carbon nanotubes (CNTs) by means of self-sustained diffusion followed by a proper rinsing. The CNTs were investigated using TEM, which showed that they were intercalated inside and clean outside. For the mixtures melting can be realized in an interval of about $20{ }^{\circ} \mathrm{C}$, which opens new opportunities for Phase Change Materials (PCM) attracting attention in relation to solar energy storage, cooling of power electronic devices and the energy-saving technologies.

* To whom correspondence should be addressed.

E-mail: ayarin@uic.edu; Phone: +1(312) 996-3472. Fax: +1(312) 413-0447. 


\section{Introduction}

Phase Change Materials (PCM) recently attracted significant attention in relation to solar energy storage $^{1-3}$, cooling of power electronic devices ${ }^{4}$ and the energy-saving technologies ${ }^{5,6}$. Different PCM were explored, e.g. ice slurry in chilled water, solid hexadecane, $n$-eicosane and paraffin waxes $^{7-9}$. Among them different combinations of paraffins seem to be the best possible alternative for PCM due to the melting temperatures of the individual paraffins being distributed over a wide temperature range, high heat of fusion, nontoxicity, stability and resistivity to corrosion. However, the organic PCM met with several problems: (i) each of these materials taken separately undergoes phase transition in a narrow temperature range; (ii) due to their very low thermal conductivity, their thermal response time might be too long; (iii) they solidify on heat exchanger surfaces. A partial remedy to problem (ii) might be in the encapsulation in PCM of copper nanoparticles ${ }^{7}$ or impregnating porous materials ${ }^{10}$, albeit an ultimate simultaneous solution of all these problems lies within nano-encapsulation of PCM. Here we explore this strategy. Specifically, we use a recently discovered method of CNT intercalation based on self-sustained diffusion ${ }^{11,12}$ applying it to different paraffins and triglycerides.

\section{Experimental}

\subsection{Materials}

Two paraffin waxes, wax $\mathrm{W} 1$, with melting point of $44-46{ }^{\circ} \mathrm{C}$, and wax $\mathrm{W} 2$, with melting point of 53$57{ }^{0} \mathrm{C}$ (the average $\mathrm{M}_{\mathrm{w}} \sim 400 \mathrm{Da}$ ) and household butter (B1, the average $\mathrm{M}_{\mathrm{w}} \sim 300 \mathrm{Da}$ ) were dissolved in benzene as described below. Commercial, as-grown, highly graphitic carbon nanotubes (PR-24, Pyrograph III) constitute the CNTs employed in this study. They contained some iron $(<14,000 \mathrm{ppm})$, and less than 5\% moisture, and had the inner diameters close to 50-100 nm and lengths in the range 10$100 \mu \mathrm{m}$.

\subsection{Solution Preparation}

Five different solutions were prepared using benzene as a solvent. Solution A contained W1 ; solution B- W2; solution C - a 1:1 (w/w) blend of W1 and W2; solution D- a 2:1 (w/w) blend of W1 and B and 
solution E- a 2:1 (w/w) blend of W2 and B. In all these cases the overall amount of solute was kept equal to $0.3 \mathrm{~g}$ per $20 \mathrm{~g}$ of solvent. After the addition of the solutes to solvents, they were sonicated for 35-40 min until a clear solution was prepared.

\subsection{Intercalation}

For the intercalation of the solutes into CNTs, 0.018- 0.022 $\mathrm{g}$ of CNTs was added to each solution and sonicated for $30 \mathrm{~min}$, which resulted in homogeneous suspensions. After that, the vial cap was kept open in a chemical hood until complete evaporation of benzene. During this process the self-sustained diffusion $^{11,12}$ of solutes into CNTs took place (as discussed below). Then, benzene was added to the deposit and sonicated for 3-4 min to rinse any solute which possibly could have been deposited outside the CNTs. It is emphasized that if sonication was continued longer than 4 min, some solute could be already lost from inside the CNTs. Following sonication, the dispersion was filtered through a syringe filter. After that, fresh benzene was passed 2-3 times to rinse any residual solute deposited on the outside of the CNTs.

\subsection{Observations}

All observations were done using JEOL JEM 3010 Transmission Electron Microscopy (TEM). Prior to the TEM observations, the samples were dried for 2-3 days to eliminate any traces of benzene.

\subsection{Differential Scanning Calorimetry}

All DSC measurements were done using DSC-Q200 by TA Instruments. Prior to the DSC measurements, all the samples were dried for 2-3 days to eliminate any traces of benzene. It is emphasized that DSC measurements were done using samples from the same batch from which samples for TEM observations were prepared.

\section{Results and Discussions}

According to Refs. 11 and 12, the intercalation of solutes (which might be molecular dispersions as in the present case, or particulates) is driven by diffusion through solvent bulk. However, in distinction 
from the ordinary diffusion where a solute content equilibration is rapidly achieved everywhere, in the case of self-sustained diffusion the simultaneous solvent evaporation permanently sustains solute gradient between the solution bulk and the inner space of CNTs. That leads to a practically $100 \%$ intercalation of CNTs with chosen solutes at room temperature and normal pressure. The process is fully scalable and transformative to the industrial applications.

Figures 1 A-E show several TEM micrographs of the intercalated CNTs densely filled inside and clean outside. Holey carbon grids were used for support, and all the observations were done with the CNT sections freely suspended over the grid holes. Thus, we avoided the interference with the imaging of any residual materials which could be present at the holey carbon surface. A careful examination of the images in Fig. 1 reveals that some ordered striations (marked by arrows; several of them highlighted in Figure 1C) are visible in the intercalated deposits inside the CNTs. As Fig. 2 shows, the inner surface of these CNTs is rather rough, which probably makes wax deposition non-uniform and maybe even unstable according to the mechanism described in Ref. 13.

The contrast of deposits in TEM micrographs is mostly determined by the molecular weights. This makes impossible to distinguish W1, W2 and B in Figs. 1 D-E. It is emphasized that paraffin waxes W1 and W2 represent themselves conglomerates of different alkanes $\left(\mathrm{C}_{n} \mathrm{H}_{2 n+2}\right.$, with $\left.20 \leq n \leq 40\right)$, whereas butter $\mathrm{B}$ is mostly triglycerides $\left(\mathrm{C}_{\mathrm{n}} \mathrm{H}_{2 \mathrm{n}-2} \mathrm{O}_{2}\right.$, with $\left.\mathrm{n}=14-20\right)$. Therefore, the only difference between W1 and W2 on the one hand, and B on the other hand, involves the presence of oxygen, which is impossible to quantify conclusively even using the Energy Dispersive X-ray (EDX) patterns in the present case. Nevertheless, the presence of $\mathrm{W} 1$ and B, as well as W2 and B in the intercalated deposits in Figs. 1D-E was fully elucidated by means of Differential Scanning Calorimetry (by using DSC-Q200, TA Instruments). For all the DSC measurements the samples were cooled to $20{ }^{0} \mathrm{C}$, then they were heated up to $80{ }^{\circ} \mathrm{C}$ at a ramp rate of $5{ }^{\circ} \mathrm{C} / \mathrm{min}$, held at $80{ }^{\circ} \mathrm{C}$ for $5 \mathrm{~min}$, and then back to $20{ }^{0} \mathrm{C}$ at a ramp rate of $5^{0} \mathrm{C} / \mathrm{min}$. 

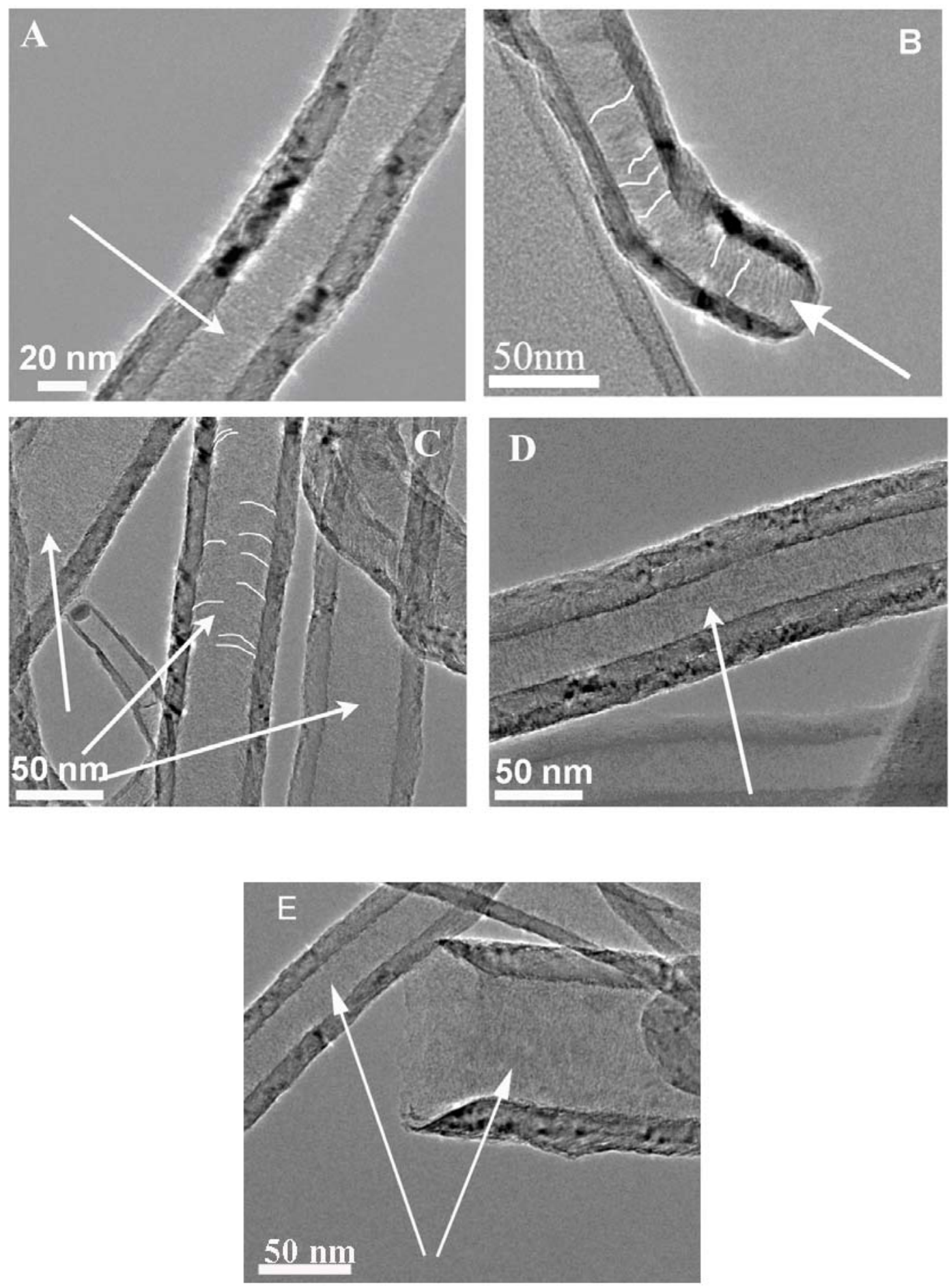

Fig. 1. TEM (JEOL JEM 3010) micrographs of carbon nanotubes intercalated by solutes A-E, respectively. The arrows point at the striations that can be seen inside the deposits in the CNTs. The solutes are deposited only inside the tubes, with no residual solutes visible outside. Panels A-E correspond to the samples intercalated with solutions A-E, respectively. Several characteristic striations are highlighted in panels $\mathrm{B}$ and $\mathrm{C}$. 


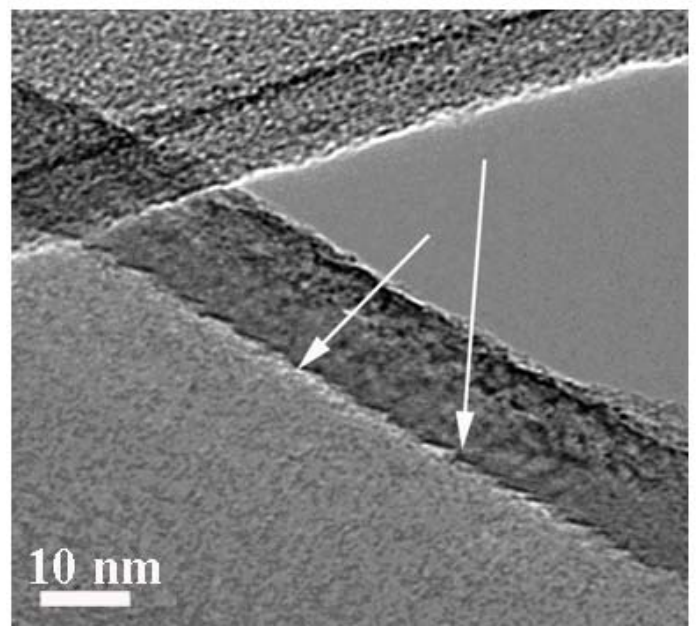

Fig. 2. TEM image of an empty CNT. The arrows point at the roughness of the inner wall.

The thermograms of bulk samples of W1, W2 and B separately were measured first (Fig. 3). The thermograms reveal that $\mathrm{W} 1$ has glass transition temperature $\left(\mathrm{T}_{\mathrm{g}}\right)$ and melting point $\left(\mathrm{T}_{\mathrm{m}}\right)$ close to $27{ }^{0} \mathrm{C}$ and $46.2{ }^{0} \mathrm{C}$, respectively, whereas for $\mathrm{W} 2$ they are $36{ }^{\circ} \mathrm{C}$ and $56{ }^{0} \mathrm{C}$, respectively. The latent heat of melting of W1 and W2 was close to $140-141 \mathrm{~J} / \mathrm{g}$ and $144-148 \mathrm{~J} / \mathrm{g}$, respectively. On the other hand, in the temperature range studied, butter revealed only one endothermic peak close to $24{ }^{0} \mathrm{C}$, which corresponds to its melting point, with the latent heat of melting being $\sim 2 \mathrm{~J} / \mathrm{g}$. 

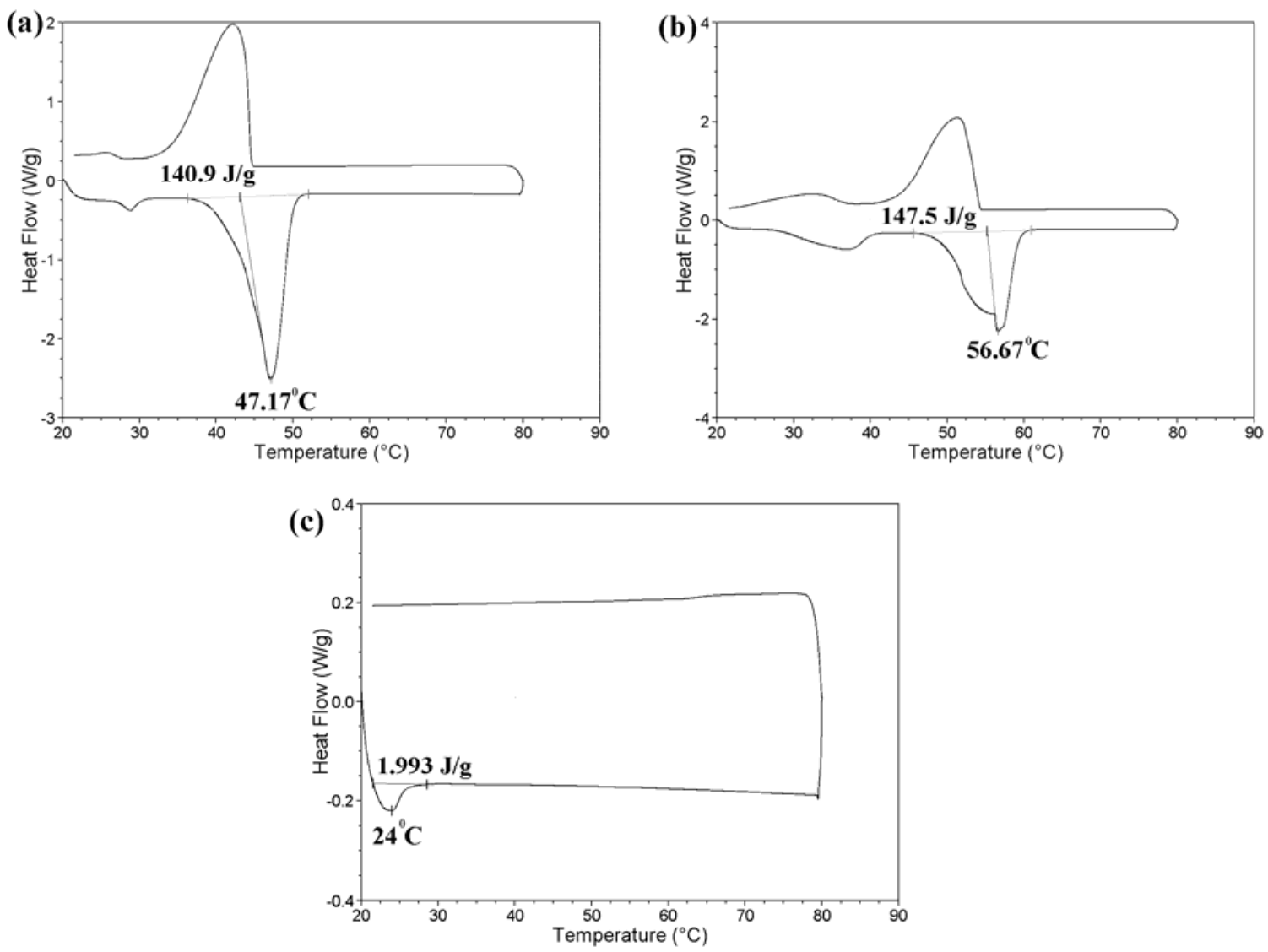

Fig. 3. Thermograms of bulk samples of (a) W1, (b) W2 and (c) B.

Then DSC of the intercalated samples of Fig. 1 was performed next. In each case samples from three different locations were used for control. The corresponding thermograms are shown in Figs. 4-6. Comparing the thermograms for bulk samples of W1 (Fig. 3 a) with that of W1 encapsulated inside CNT (Fig. 4a), one can observe that in the latter case the endothermic peak corresponding to the glass transition temperature of the bulk sample disappeared. Moreover, the melting point of the bulk sample of $\mathrm{W} 1$ of $47.17{ }^{0} \mathrm{C}$ (Fig. 3a) is replaced by $44.24{ }^{0} \mathrm{C}$ for the intercalated W1 (Fig. 4a). A similar trend was found for W2: the bulk melting point of $56.67{ }^{0} \mathrm{C}$ (Fig. 3b) was shifted to $52.64{ }^{0} \mathrm{C}$ for the intercalated sample (Fig. 4b). The thermograms show that for W2 the glass transition in the temperature range studied has not disappeared completely, albeit deteriorated significantly. The reduction of the melt temperature in the intercalated samples is attributed to changes in the confined crystalline structure formed inside $\mathrm{CNT}^{12}$. 
Figures $5 \mathrm{a}$ and $\mathrm{b}$ correspond to the intercalated blends of $\mathrm{W} 1$ and $\mathrm{B}$, as well as $\mathrm{W} 2$ and $\mathrm{B}$ (both in the 2:1 ratio). They reveal heat absorption in a much wider temperature range than that for pure $\mathrm{W} 1$ and W2. Indeed, W1 and B revealed heat absorption in the range of $28{ }^{0} \mathrm{C}-52{ }^{0} \mathrm{C}$ (Fig. 5a) versus that of pure $\mathrm{W} 1$ only in the range of $35{ }^{\circ} \mathrm{C}-52{ }^{0} \mathrm{C}$ in Fig. 3a. Similarly for W2 and B in CNTs in Fig. $5 \mathrm{~b}$ the heat absorption range of $26{ }^{0} \mathrm{C}-62{ }^{0} \mathrm{C}$ is wider than for pure $\mathrm{W} 2-36{ }^{0} \mathrm{C}-62{ }^{0} \mathrm{C}$ in Fig. $4 \mathrm{~b}$. In addition, the intercalated blend samples of two different waxes, W1 and W2, demonstrate the heat absorption range of $35{ }^{0} \mathrm{C}-62{ }^{0} \mathrm{C}$ (Figure 6), which is an overlap of the heat absorption ranges of these waxes separately (Figs. 4 a and b). All these results show that the temperature range of PCM heat absorption can be tuned by tailoring the composition of the intercalated materials in CNTs. This effectively solves problem (i) mentioned above. In addition, it should be noted that the intercalated B occupies some space and makes wax confinement even smaller, which can affect and change its crystalline structure even more. This, in turn, determines its melting in a wider temperature "window".
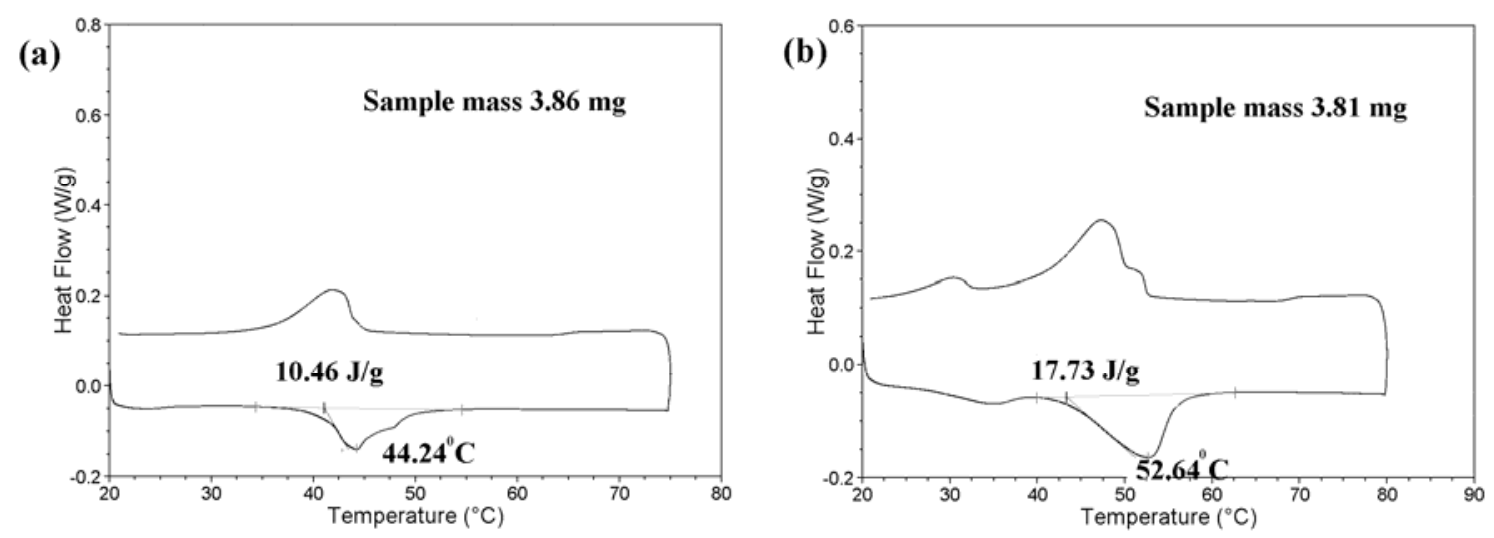

Fig. 4. Thermograms of the intercalated wax in CNT: (a) Pure W1; and (b) Pure W2. 
(a)

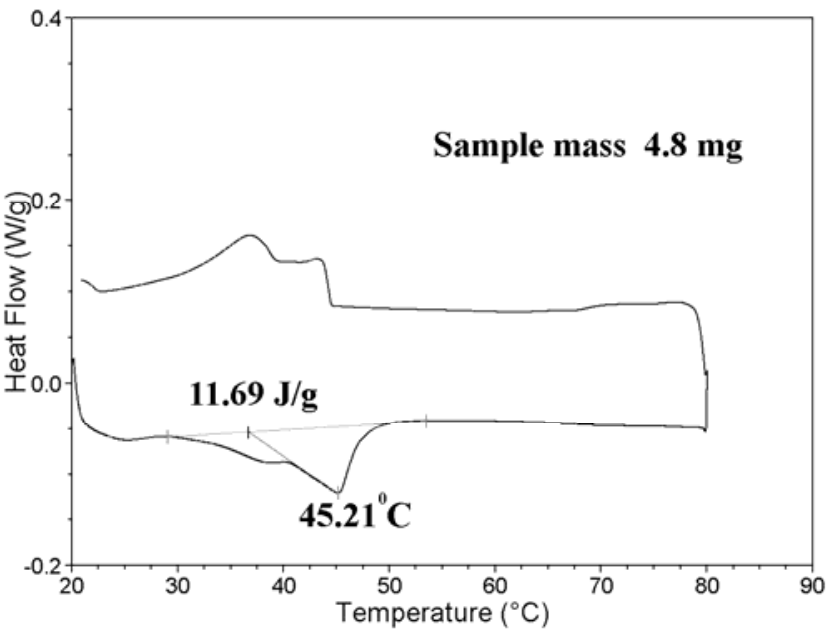

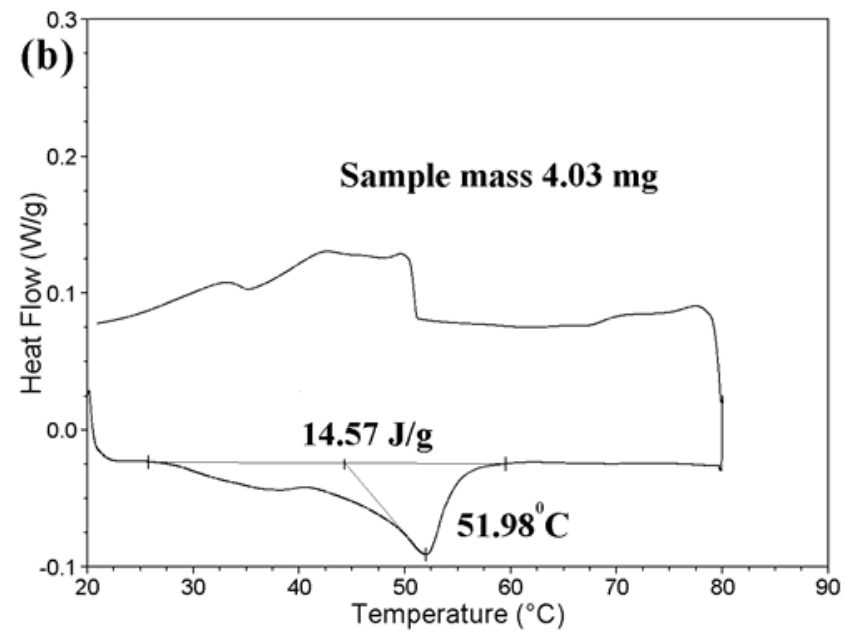

Fig. 5. Thermograms of the intercalated blended deposits in CNT: (a) 2:1 blend of W1 and B; (b) 2:1 blend of $\mathrm{W} 2$ and $\mathrm{B}$.

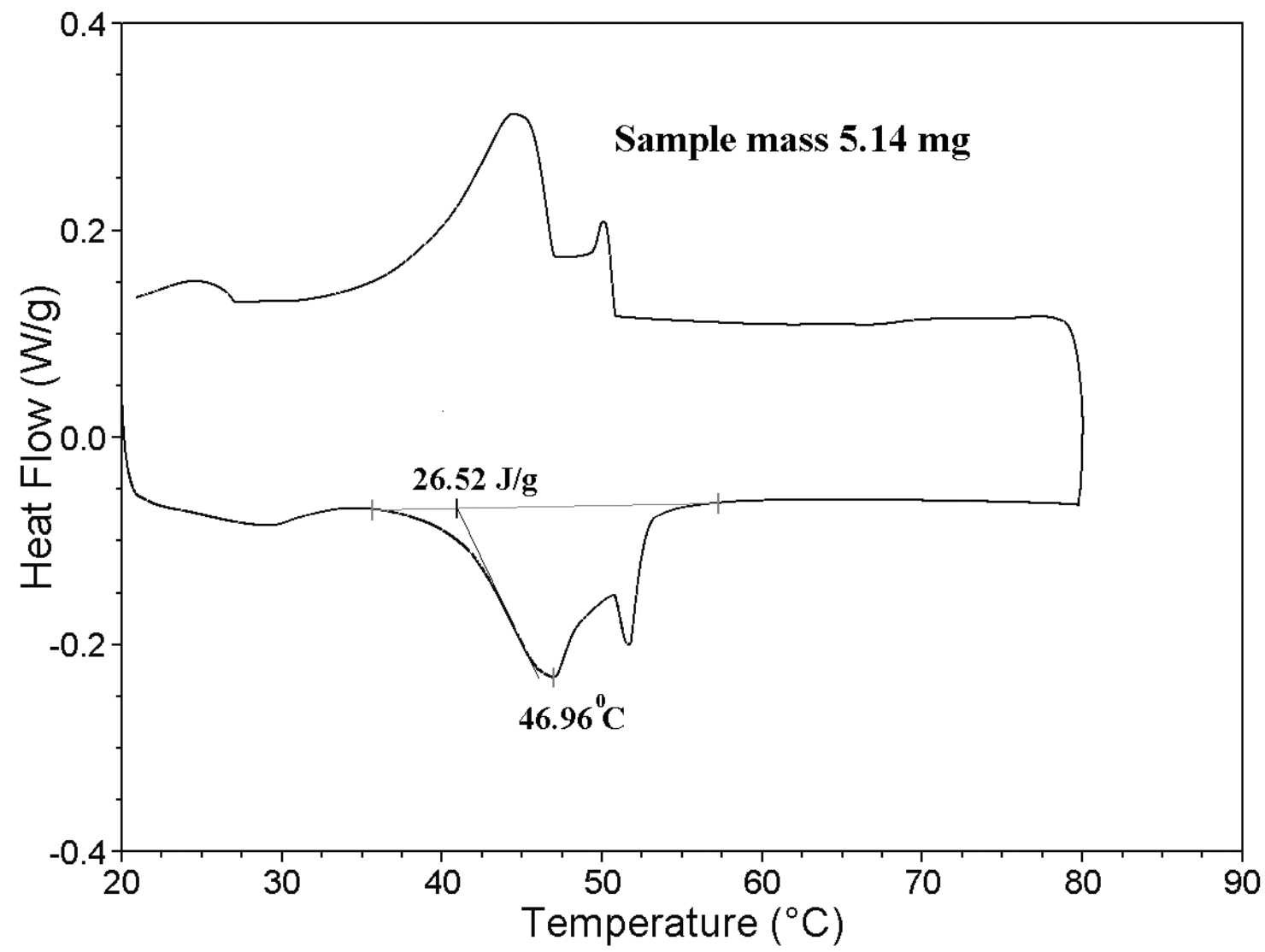

Fig. 6. Thermogram of 1:1 blend of $\mathrm{W} 1$ and $\mathrm{W} 2$ intercalated in CNT. 
The latent heat of melting is known for both $\mathrm{W} 1$ and W2. Their molecular weights are approximately the same. Then, the thermogram in Fig. 6 can be used to evaluate the masses of W1 and W2 intercalated into a given CNT sample. As a result, we find that in the case of a 1:1 blend of W1 and W2, the intercalated overall mass was close to $20 \%$ of the encasing CNTs mass. Moreover, the proportion of W1 to $\mathrm{W} 2$ in the original solution $(\mathrm{C})$ was practically replicated into the composition inside CNTs, since with the close molecular weights, molecules of these two waxes had close gyration radii, and thus close diffusion rates ${ }^{11}$. Therefore, the masses of $\mathrm{W} 1$ and $\mathrm{W} 2$ in CNTs were approximately $10 \%$ each of the mass of the encasing CNTs.

However, W1 (or W2) and B had different molecular weights, so one cannot expect that their proportion in a binary solution $(\mathrm{W} 1+\mathrm{B}$ or $\mathrm{W} 2+\mathrm{B})$ will be replicated in the intercalated deposit. On the other hand, the latent heat of melting of B is negligibly small compared to that of W1 or W2. Therefore, the thermograms of Fig. 5 reveal the intercalated masses of W1 or W2 directly. This shows that for the intercalated samples from solutions D-E the amount of wax inside CNTs is of the order of $8.8 \%$ and $11.08 \%$ of the mass of the CNTs samples, respectively.

The thermal diffusivity $\alpha$ of wax is known to be of the order $10^{-7} \mathrm{~m}^{2} / \mathrm{s}{ }^{14}$. Taking for the estimate the average $\mathrm{CNT}$ diameter $\mathrm{D}$ of the order of $100 \mathrm{~nm}$, we estimate the characteristic thermal response time as $\mathrm{D}^{2} / \alpha \sim 10^{-7} \mathrm{~s}$. This shows that the thermal response time of a single nano-encapsulated PCM particle is immaterial, which effectively solves problem (ii) mentioned above and eliminates the need in the intercalation of copper nanoparticles. The heating time of a liquid coolant carrying nano-encapsulated PCM particles is of the order of $\mathrm{L}^{2} / \alpha_{\mathrm{c}}$ where $\mathrm{L}$ is the characteristic volume size and $\alpha_{\mathrm{c}}$ is the thermal diffusivity of the coolant. The PCM nanoparticles will have enough time for the phase transition in flow near a hot element, if $\mathrm{L}^{2} / \alpha_{\mathrm{c}}$ is the order of the characteristic flow time $\mathrm{L} / \mathrm{V}$ with $\mathrm{V}$ being the flow velocity, since the particle phase transition time is immaterial. This recast into the requirement that the Peclet number $\mathrm{Pe}=\mathrm{LV} / \alpha_{\mathrm{c}} \sim 1$. Taking for the estimate flows of typical coolants (e.g. water or refrigerant HFC-22) in microchannels, we have $\mathrm{L} \sim 100 \mu \mathrm{m}, \mathrm{V} \sim 1 \mathrm{~mm} / \mathrm{s}$ and $\alpha_{\mathrm{c}} \sim 10^{-7} \mathrm{~m}^{2} / \mathrm{s}$, which yields 
Pe 1. Therefore, PCM nanoparticles will have enough time to be molten and adsorb heat in the form of the latent heat of phase transition.

Since the encapsulated wax cannot solidify on heat exchanger surfaces, problem (iii) can also be effectively eliminated.

\section{Conclusion}

We have demonstrated a novel technique to intercalate paraffin waxes, as well as their blends with triglycerides inside carbon nanotubes using self-sustained diffusion. The nano-scale phase change materials (PCM) obtained using this route can be tailored to possess a relatively wide working temperature range, as well as a negligible thermal response time. The method allows a straightforward scale up and can be used for mass production of PCM for solar energy storage, cooling of power electronic devices and the energy-saving technologies.

\section{Acknowledgement}

This research was partially supported by DOE in the framework of the project "Filled Carbon Nanotubes: Superior Latent Heat Storage Enhancers”.

\section{References}

1. L. M. Bal, S. Satya, S. N. Naik and V. Meda, Renew. Sustain. Energy Rev., 2011, 15, 876-880.

2. M. A. Hossain, J. L. Woods and B. K. Bala, Renew. Energy, 2005, 30, 729-742.

3. Y. Zhiqiang, Solar Energy Materials \& Solar Cells, 2005, 86, 427-442.

4. R. Sabbah, M. M. Farid and S. Al-Hallaj, Appl. Therm. Eng., 2009, 29, 445-454.

5. P. Schossig, H. M. Henning, S. Gschwander and T. Haussmann, Solar Energy Materials \& Solar Cells, 2005, 89, 297-306. 
6. F. Kuznik, D. David, K. Johannes and J. J. Roux, Renew. Sustain. Energy Rev., 2011, 15, 379-391.

7. S. Wu, D. Zhu, X. Zhang and J. Huang, Energy Fuels, 2010, 24, 1894-1898.

8. F. Agyenim, N. Hewitt, P. Eames and M. Smyth, Renew. Sustain. Energy Rev., 2010, 14, 615-628.

9. M. Akgun, O. Aydın and K. Kaygusuz, Appl. Therm. Eng., 2008, 28, 405-413.

10. A. Karaipekli and A. Sari, Sol. Energy, 2009, 83, 323-332.

11. A. V. Bazilevsky, K. Sun, A. L. Yarin and C. M. Megaridis, Langmuir, 2007, 23, 74517455.

12. A. V. Bazilevsky, K. Sun, A. L. Yarin and C. M. Megaridis, J. Mater. Chem., 2008, 18, 696-702.

13. R. Srikar, A. L. Yarin and C. M. Megaridis, Nanotechnology., 2009, 20, 275706.

14. G. E. Totten, S. R. Westbrook and R. J. Shah, Fuels and Lubricants Handbook: Technology, Properties, Performance and Testing, Vol. 1. ASTM International: West Conshocken, PA, 2003. 